AperTO - Archivio Istituzionale Open Access dell'Università di Torino

\title{
A Fast Chromatographic Method for Estimating Lipophilicity and Ionization in Nonpolar Membrane-Like Environment
}

\section{This is the author's manuscript}

Original Citation:

Availability:

This version is available http://hdl.handle.net/2318/1571833

since 2016-06-24T17:11:42Z

Published version:

DOI:10.1021/acs.molpharmaceut.5b00910

Terms of use:

Open Access

Anyone can freely access the full text of works made available as "Open Access". Works made available under a Creative Commons license can be used according to the terms and conditions of said license. Use of all other works requires consent of the right holder (author or publisher) if not exempted from copyright protection by the applicable law. 
This is the author's final version of the contribution published as:

Caron, Giulia; Vallaro, Maura; Ermondi, Giuseppe; Goetz, Gilles H.; Abramov, Yuriy A.; Philippe, Laurence; Shalaeva, Marina. A Fast Chromatographic Method for Estimating Lipophilicity and Ionization in Nonpolar Membrane-Like Environment. MOLECULAR

PHARMACEUTICS. 13 (3) pp: 1100-1110.

DOI: 10.1021/acs.molpharmaceut.5b00910

The publisher's version is available at:

http://pubs.acs.org/doi/abs/10.1021/acs.molpharmaceut.5b00910

When citing, please refer to the published version.

Link to this full text:

http://hdl.handle.net/2318/1571833 


\section{A Fast Chromatographic Method for Estimating Lipophilicity and Ionization in Nonpolar Membrane-Like Environment}

Giulia Caron ${ }^{1}$, Maura Vallaro ${ }^{I}$, Giuseppe Ermondi, Gilles H. Goetz ${ }^{2}$, Yuriy A. Abramov ${ }^{3}$, Laurence Philippe $^{2}$, Marina Shalaeva ${ }^{2} *$

${ }^{1}$ Molecular Biotechnology and Health Sciences Dept., Università degli Studi di Torino, via Quarello 15, 10135 Torino, Italy.

${ }^{2}$ Worldwide Medicinal Chemistry, Pfizer Worldwide Research \& Development, Pfizer, Inc., Groton, Connecticut 06340, United States

${ }^{3}$ Worldwide Pharmaceutical Sciences, Pfizer Worldwide Research \& Development, Pfizer, Inc., Groton, Connecticut 06340, United States

* Pfizer Worldwide Research \& Development, Pfizer, Inc.,

Eastern Point Road, Groton, CT 06340, United States

Tel 860 441-0569, Fax 860 715-3345

Email: marina.shalaeva@pfizer.com 
Table of Contents Graphic

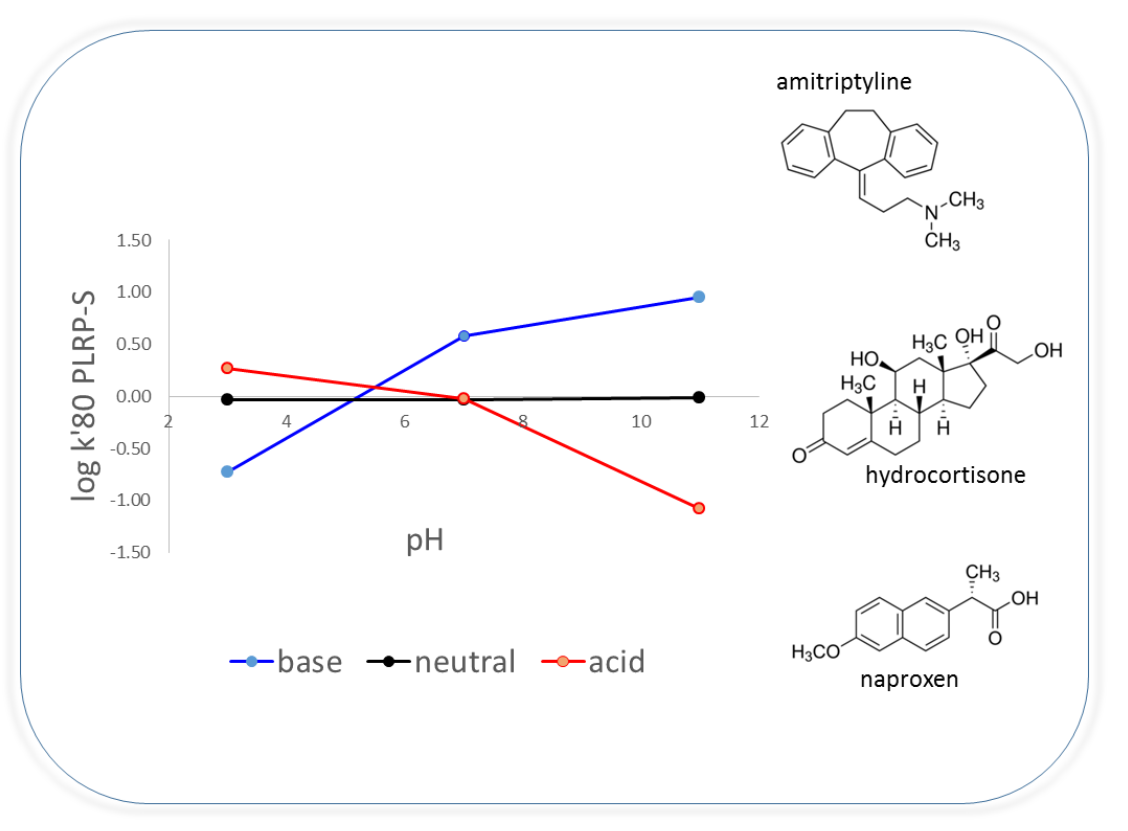




\section{Abstract}

This study describes the design and implementation of a new chromatographic descriptor called log k' 80 PLRP-S that provides information about the lipophilicity of drug molecules in nonpolar environment, both in their neutral and ionized form.

The log k'80 PLRP-S obtained on a polymeric column with acetonitrile/water mobile phase is shown to closely relate to $\log \mathrm{P}_{\text {toluene }}$ (toluene dielectric constant $\varepsilon \sim 2$ ).

The main intermolecular interactions governing log k'80 PLRP-S were deconvoluted using the Block Relevance (BR) analysis. The information provided by this descriptor compared to ElogD and calclog $\mathrm{P}_{\text {tol }}$ and the differences are highlighted.

The "charge-flush" concept is introduced to describe the sensitivity of log k' 80 PLRP-S to ionization state of compounds in $\mathrm{pH}$ range 2 to 12 .

The ability of log k'80 PLRP-S to indicate the propensity of neutral molecules and monoanions to form Intramolecular Hydrogen Bonds (IMHBs) is proven through a number of examples.

\section{Keywords}

Nonpolar environments, Block Relevance analysis, drug candidates, ElogD, $\log \mathrm{P}_{\mathrm{tol}}, \log$ k'80 PLRP-S, lipophilicity.

\section{Abbreviations}

BR: Block Relevance

COSMO-RS: COnductor like Screening MOdel for Real Solvents

IMHB: Intramolecular Hydrogen Bonding

PCA: Principal Component Analysis

PLS: Partial Least Squares

PLRP-S: polystyrene/divinylbenzene polymeric column

QSAR: Quantitative Structure-Activity Relationships

QSPR: Quantitative Structure-Property Relationships 


\section{Introduction}

The lipophilicity descriptors determined in the $n$-octanol/water biphasic system (e.g. $\log \mathrm{P}_{\text {oct }}$ and $\log \mathrm{D}_{\text {oct }}$ ) are routinely used by medicinal chemists to optimize the drug-like properties of hits and leads in the various steps of the drug discovery process. ${ }^{1}$ However, many interactions by the drug molecules are taking place in multilayered compartments, such as biological membranes. There is a need to support $\log \mathrm{P}_{\text {oct }}$ and $\log \mathrm{D}_{\text {oct }}$ with additional indexes that describe the affinity of molecules for specific physiological environments. For example, the central portion of the biomembranes, characterized by low dielectric constant $(\sim 2), 2,3$ cannot be adequately modeled by $n$-octanol (dielectric constant $\sim 10$ ). The lipophilicity descriptors which could characterize the compound properties in the core of biological membranes are expected to be of great relevance to drug discovery programs. ${ }^{4}$

The most common descriptor to evaluate lipophilicity in nonpolar environments is $\log \mathrm{P}_{\text {alk. }}$ Unfortunately, this descriptor is largely inaccessible for many drug candidates due to their poor solubility in the alkane phase. The $\log \mathrm{P}_{\text {tol }}$ has been recently proposed as a valuable alternative ${ }^{5}$ to $\log \mathrm{P}_{\text {alk }}$ due to better solubility in toluene. Moreover, we have tested COSMO-RS software and demonstrated that it is a reliable tool for log $\mathrm{P}_{\text {tol }}$ prediction. ${ }^{5}$ Nevertheless, the "shake-flask" methodology, in general, is limited by the difficulty of quantification in organic or water phases, especially for $-3<\log$ P $>3$. HPLC-based methods ${ }^{6}$ have helped to overcome the limitations stated above and have gained broad acceptance for lipophilicity assessment.

Indeed, chromatographic systems can be easily set-up with a variety of available stationary and mobile phases to reproduce a number of biological environments. ${ }^{7}$ For instance, ElogD is a widely accepted RPHPLC method for $\log \mathrm{D}_{\text {oct }}$ determination; ${ }^{8}$ and EPSA ${ }^{9}$ is a Supercritical Fluid Chromatography (SFC) method describing exposed polarity of the molecule in low dielectric constant environment. A few studies have been published over the years suggesting that the polymeric columns ${ }^{10,11,12,13}$ could be useful for characterization of drug candidates.

In this study we describe a chromatographic system with polystyrene/divinylbenzene polymeric column (PLRP-S) and introduce the lipophilicity index based on retention in that system called log k'80 PLRP-S, where 80 is referring to the percentage of the aprotic acetonitrile in the mobile phase.

In order to characterize the system we applied the recently developed Block Relevance (BR) analysis tool, ${ }^{14}$ which allowed the analysis of the balance of intermolecular interactions governing the retention using common 3D-QSAR/QSPR descriptors. These descriptors are aggregated into property-related groups (blocks), thus providing a convenient framework for comparison and interpretation of descriptors determined in different systems, for example ElogD, vs. $\log \mathrm{P}_{\text {tol }}$, vs. $\log$ k'80 PLRP-S. Furthermore, the stability of PLRP-S polymeric column under a wide range of $\mathrm{pH}$ conditions $(1-12)$ is an important feature of this type of stationary phase and we wanted to explore its advantages for characterizing ionizable compounds.

The goals of this study can be summarized in three areas:

1. Establish the relationship between $\log \mathrm{k}^{\prime} 80$ PLRP-S and other lipophilicity descriptors (ElogD ${ }^{8}$ and calclog $\mathrm{P}_{\text {tol }}{ }^{5}$ ) using a representative set of neutral compounds, including common drugs. 
2. Investigate the sensitivity of the new descriptor to compounds propensity to form intramolecular hydrogen bonds (IMHBs). The log k'80 PLRP-S values were compared for the pairs of structural analogs capable and not-capable of IMHB, similar to the previously established $\Delta \log$ P approach, ${ }^{12}$ and the capabilities for IMHB detection are reported.

3. Verify that the system provides information on the state of ionization of compounds at different pHs. Here a fairly large set of ionizable commercial drugs was assembled, for which we determined and analyzed log k'80 PLRP-S at acidic, neutral and basic pHs. The unique feature of the PLRP-S system to relate the ionization state of the molecules in a low dielectric constant environment, similar to biomembranes, is identified and described.

Taken together, these results suggest that log k'80 PLRP-S is a descriptor that allows estimation of lipophilicity of neutral and ionized species in nonpolar matrix and deserve being included in drug discovery strategies.

\section{Experimental Section}

\section{The datasets}

The selection of the panel of compounds to investigate and set-up a new method to be integrated in drug discovery programs is not a trivial issue. Simple organic compounds are very helpful for the mechanistic interpretation of the phenomenon, but they cover a limited chemical space. Commercial drugs, on the other hand, enable the enlargement of the chemical space and thus should be included. Furthermore, the internal data sets are required to demonstrate the practical application of the method to the chemical space explored by the current drug design projects.

The first dataset was comprised of 44 neutral compounds, which includes simple organic molecules, commercial drugs and internal compounds (Table 1). Chemical structures can be downloaded from https://sites.google.com/site/cassmedchem/download-area/plrps-dataset.

None of these compounds have any propensity to form IMHBs and are predominantly neutral at $\mathrm{pH}$ 7. The dataset diversity was explored using Principal Component Analysis (PCA). The scores and loadings plots resulting from PCA analysis using 82 VolSurf+ descriptors are reported in Figures S1A and S1B (Supporting Information), respectively. In order to check for the diversity of the chemical space covered by this dataset we made a comparison with the Skold's dataset, ${ }^{15}$ which was proposed as a gold standard for physicochemical pharmaceutical profiling. The PCA analysis showed that the chemical space covered by the 44 neutral compounds was comparable to Skold's dataset (data not shown).

The second dataset consists of 5 pairs (10 compounds) capable and incapable of forming IMHBs. ${ }^{5}$ The chemical structures are shown in Figure 3.

The third dataset was composed of 76 commercial drugs (Table 3). This dataset includes drugs in various states of ionization and a wide range of $\mathrm{pK}_{\mathrm{a}}$ values. Molecular properties were calculated (e.g. $\log \mathrm{D}^{7.4}$, number of HBD/HBA groups, rotatable bonds, etc.) to assess the chemical diversity of compounds present in 
the dataset (data not shown). Chemical structures can be downloaded from https://sites.google.com/site/cassmedchem/download-area/plrps-dataset.

\section{Chromatographic measurements}

\section{$\log k^{\prime} 80$ PLRP-S}

Measurements were performed using an Agilent 1290 HPLC instrument with diode array and quadrupole mass-spec 6140 detectors. The UV signals were monitored at 215, 254, 280 and $310 \mathrm{~nm}$ and MS detection by ES API in positive mode. The retention time from the UV signal was used throughout for consistency, unless noted. The PLRP-S column, 100 A, 5 uM, 50x4.6mm; part\# PL1512-1500 from Agilent was used with the mobile phase flow rate $1 \mathrm{~mL} / \mathrm{min}$.

The mobile phase was prepared by mixing $80 \%$ acetonitrile and $20 \%$ of aqueous portion, which contained the total amount of the buffer. The aqueous buffers were chosen to cover $\mathrm{pH}$ range from 2 to 11 and also to be compatible with mass-spec detection: $0.1 \%$ formate buffer; $0.1 \%$ ammonium acetate and $10 \mathrm{mM}$ triethylamine.

Astemizole was used as $\mathrm{t}_{0}$ marker $(\mathrm{RT}=0.3 \mathrm{~min}$.) at $\mathrm{pH} 2$ and valsartan $(\mathrm{RT}=0.29 \mathrm{~min}$.) at $\mathrm{pH} \sim 10$. We used $\mathrm{t}_{0}=0.3 \mathrm{~min}$. throughout for consistency in calculations of the capacity factor $\mathrm{k}$.

The log k'80 PLRP-S standard deviation determined on two columns on a set of 49 compounds form the first and second datasets, measured 3 times, was 0.021 .

ElogD

ElogD measurements were performed as previously described. ${ }^{8}$ Briefly, ElogD values obtained from $\operatorname{logk}{ }^{\prime} w$ determined on Supelcosil LC-ABZ column using MOPS buffer and methanol mobile phase at pH 7.4.

\section{NMR measurements}

The proton chemical shift ${ }^{1} \mathrm{H}$ NMR values used here were determined in $\mathrm{CDCl}_{3}$ and DMSO- $d 6$ as previously described. ${ }^{5,16}$

\section{pH measurements}

Agilent 3200P pH meter and P3211 combination electrode calibrated with aqueous standards were used for $\mathrm{pH}$ measurements. The $\mathrm{pH}$ of the aqueous portion of the mobile phase was measured and recorded, and then $80 \%$ of acetonitrile added. The ${ }^{\mathrm{s}} \mathrm{pH}(\mathrm{pH}$ measured in acetonitrile/water with the electrode calibrated in water) of the mobile phase was measured after the mixture equilibrated to the room temperature.

\section{In silico tools}

\section{Propensity to form IMHBs}

An in-house software ${ }^{17}$ was used to identify compounds with propensity to form intramolecular hydrogen bonds (IMHB) according to the topologies proposed by Kuhn and coworkers. ${ }^{18}$

\section{$\mathrm{pK}_{\mathrm{a}}$ calculations}

$\mathrm{pK}_{\mathrm{a}}$ calculations were performed using MoKa (Molecular Discoveries, Ltd, v.2.5.4 ) and ACDlabs Percepta v. 12.1 . 


\section{COSMO-RS calculations of $\log \mathbf{P}_{\text {tol }}$ data}

$\log \mathrm{P}_{\text {tol }}$ calculations were performed using COSMO-RS software as previously described. ${ }^{5}$

VS+ models

VS+ models were built by submitting the SMILES codes of the compounds to VS+ (version 1.0.7, http://www.moldiscovery.com) using default settings and four probes (OH2, DRY N1 and O probes that mimic respectively water, hydrophobic, HBA and HBD properties of the environment). PCA and PLS tools implemented in VS+ were used.

\section{$\mathrm{BR}$ analysis}

BR analysis was performed as described elsewhere. ${ }^{14,17}$ Briefly, BR analysis allows useful interpretation of PLS models by organizing the VS+ descriptors into six blocks (Size, Water, DRY, N1, O and Others) of straightforward significance. The definitions and details are given in Figure 1B. In a very simplistic way, BR analysis gives the relevance of each parameter (block) to the model.

Processing was done on two 8 cores Xeon E5 at 3.3GHz CPUs and 128GB of RAM.

\section{Results}

\section{The PLRP-S system characterization}

A set of 44 compounds, mostly neutral at $\mathrm{pH}$ 7, with no propensity to form IMHBs, was selected for the comparative analysis of the chromatographic system consisting of a) a PLRP-S column made of polystyrene-divinylbenzene copolymer as a stationary phase and b) a mobile phase made by mixing $80 \%$ $\mathrm{ACN}$ and $20 \%$ aqueous buffer.

The obtained descriptor is named log k'80 PLRP-S and defined as follows:

$\log k^{\prime} 80 P L R P-S=\log \left(\left(t_{R}-t_{0}\right) / t_{0}\right)$

where $\mathrm{k}^{\prime} 80$ PLRP-S is the retention factor, $\mathrm{t}_{\mathrm{R}}$ is the retention time and $\mathrm{t}_{0}$ is the column dead time.

The full list of experimental $\log$ k' 80 PLRP-S is reported in Table 1. For comparative purposes ElogD ${ }^{8}$ and calculated $\log \mathrm{P}_{\text {tol }}{ }^{5}$ (calclog $\left.\mathrm{P}_{\text {tol }}\right)$ values are also reported here.

Table 1 reveals that log k'80 PLRP-S covers a range of 1.25 logarithmic units which is significantly smaller than those covered by $\operatorname{Elog} \mathrm{D}(5.16)$ and calclog $\mathrm{P}_{\text {tol }}$ (7.91).

Table 1. Dataset investigated for comparative characterization of PLRP-S system. The compounds listed with letters and numbers reflect the original assignments made in the previous studies. ${ }^{5,16}$

\begin{tabular}{llll}
\hline Compounds & $\log$ k'80 PLRP-S & ElogD & calclog $\mathbf{P}_{\text {tol }}$ \\
\hline 1-naphthol & 0.34 & 3.17 & 1.58 \\
2-naphthol & 0.29 & 3.01 & 1.37 \\
3,5-dichlorophenol & 0.46 & 3.88 & 1.1
\end{tabular}




\begin{tabular}{|c|c|c|c|}
\hline 3-bromoquinoline & 0.84 & 2.93 & 3.36 \\
\hline 3-chlorophenol & 0.26 & 3.11 & 0.64 \\
\hline 3-nitroaniline & 0.34 & 1.52 & 1.31 \\
\hline 3-nitrophenol & 0.24 & 2.22 & 0.2 \\
\hline 4-toluidine & 0.32 & 1.48 & 1.55 \\
\hline Acetophenone & 0.40 & 1.58 & 1.88 \\
\hline Aniline & 0.26 & 0.69 & 1.07 \\
\hline Antipyrine & -0.04 & 0.23 & 0.58 \\
\hline Bifonazole & 0.67 & 4.95 & 4.74 \\
\hline Bromazepam & 0.22 & 1.38 & 1.52 \\
\hline $\mathbf{c A}^{5}$ & 0.36 & 2.68 & 2.44 \\
\hline caffeine & -0.04 & -0.21 & -0.32 \\
\hline carbamazepine & 0.09 & 2.01 & 0.98 \\
\hline $\mathbf{c B}^{5}$ & 0.64 & 2.58 & 3.76 \\
\hline $\mathbf{c D}^{5}$ & 0.10 & 1.84 & 1.09 \\
\hline cE1 $^{5}$ & 0.16 & 2.11 & 0.99 \\
\hline $\mathbf{c F}^{5}$ & 0.05 & 1.3 & -0.43 \\
\hline $\mathbf{c H}^{5}$ & 0.36 & 1.65 & 2.69 \\
\hline clotrimazole & 0.65 & 4.69 & 4.66 \\
\hline dexamethasone & 0.10 & 2.37 & 0.76 \\
\hline diazepam & 0.50 & 3.03 & 3.34 \\
\hline diethylstilbestrol & 0.35 & 4.72 & 2.03 \\
\hline estradiol & 0.29 & 3.82 & 1.62 \\
\hline griseofulvin & 0.26 & 2.57 & 2.35 \\
\hline hydrocortisone & -0.01 & 1.71 & -0.06 \\
\hline hydrocortisone- 21 -acetate & 0.20 & 2.26 & 0.26 \\
\hline lorazepam & 0.19 & 2.74 & 1.58 \\
\hline lormetazepam & 0.34 & 2.78 & 2.91 \\
\hline naphthalene & 0.98 & 3.62 & 3.71 \\
\hline nefazadone & 1.21 & 4.95 & 6.49 \\
\hline nifedipine & 0.29 & 2.85 & 2.94 \\
\hline nifuroxime & 0.11 & 1.36 & -0.06 \\
\hline pentoxifylline & 0.09 & 0.31 & 0.2 \\
\hline $\mathbf{6}^{16}$ & 0.01 & -0.1 & -1.06 \\
\hline phenol & 0.16 & 1.49 & 0.21 \\
\hline prednisolone & 0.09 & 1.83 & -0.55 \\
\hline prednisone & 0.09 & 1.47 & 0 \\
\hline quinoline & 0.42 & 1.85 & 1.96 \\
\hline testosterone & 0.30 & 3.15 & 1.3 \\
\hline thymol & 0.38 & 3.65 & 2.75 \\
\hline tolnaftate & 1.16 & 5.15 & 6.85 \\
\hline
\end{tabular}

Data in Table 1 were used to build the first QSPR model by splitting up the dataset into a training set $(\mathrm{n}=33$ ) and a test set $(\mathrm{n}=11)$. Experimental log k'80 PLRP-S values were imported into VS+ as response variables ( $\mathrm{Y}$, the property) and a relation between $\mathrm{Y}$ and the $82 \mathrm{VS}+$ molecular descriptors (X, the structure) was sought using the PLS algorithm implemented in the software. A model was found $\left(\mathrm{R}^{2}=0.87, \mathrm{Q}^{2}=0.53\right.$, 
Table 2) and the correlation between calculated and experimental values $\left(\mathrm{R}^{2}=0.87\right)$ is shown in Figure $\mathrm{S} 2$ (Supporting Information). The external validation of the PLS model using the test set was also performed. Predictions are shown in Figure S2 $\left(\mathrm{R}^{2}=0.84\right.$, Supporting Information) and support the statistical stability of the model.

Table 2. PLS models $\left(N=\right.$ number of observations, $R^{2}=$ cumulative determination coefficient, $Q^{2}=$ crossvalidated correlation coefficient, $L V=$ number of latent variables, SDEP $=$ standard error on the prediction).

\begin{tabular}{llllll}
\hline System & $\mathbf{N}$ & $\mathbf{R}^{\mathbf{2}}$ & $\mathbf{Q}^{\mathbf{2}}$ & $\mathbf{L V}$ & SDEP \\
\hline $\log$ k'80 PLRP-S & 33 & 0.87 & 0.53 & 4 & 0.22 \\
ElogD & 33 & 0.89 & 0.67 & 4 & 0.80 \\
calclog tol & 33 & 0.90 & 0.67 & 4 & 1.04 \\
$\log$ k'80 PLRP-S & 44 & 0.86 & 0.63 & 4 & 0.17 \\
ElogD & 44 & 0.89 & 0.78 & 4 & 0.62 \\
calclog $P_{\text {tol }}$ & 44 & 0.89 & 0.74 & 4 & 0.88 \\
\hline
\end{tabular}

Since the external validation procedure gave excellent results, a second PLS model based on all 44 compounds was built (Table $2, \mathrm{R}^{2}=0.86, \mathrm{Q}^{2}=0.63$ ) and used as a starting point for the BR analysis. ${ }^{14,17,19,20,21}$ BR analysis graphical output for log k'80 PLRP-S is reported in Figure 1A. The meaning and significance of the blocks is reported in Figure 1B to help with interpretation of parameters.

Figure 1A outlines the major role played by the Size block (green) in log k' 80 PLRP-S values. Moreover, it shows that the Size block (green) and the O block (related to HBD solutes' properties) are almost entirely attributed either to positive or negative portions, respectively.

Figure 1. BR analysis graphical outputs: A) log k'80 PLRP-S, B) block significance, C) ElogD and D) calclog $P_{t o l}$ 

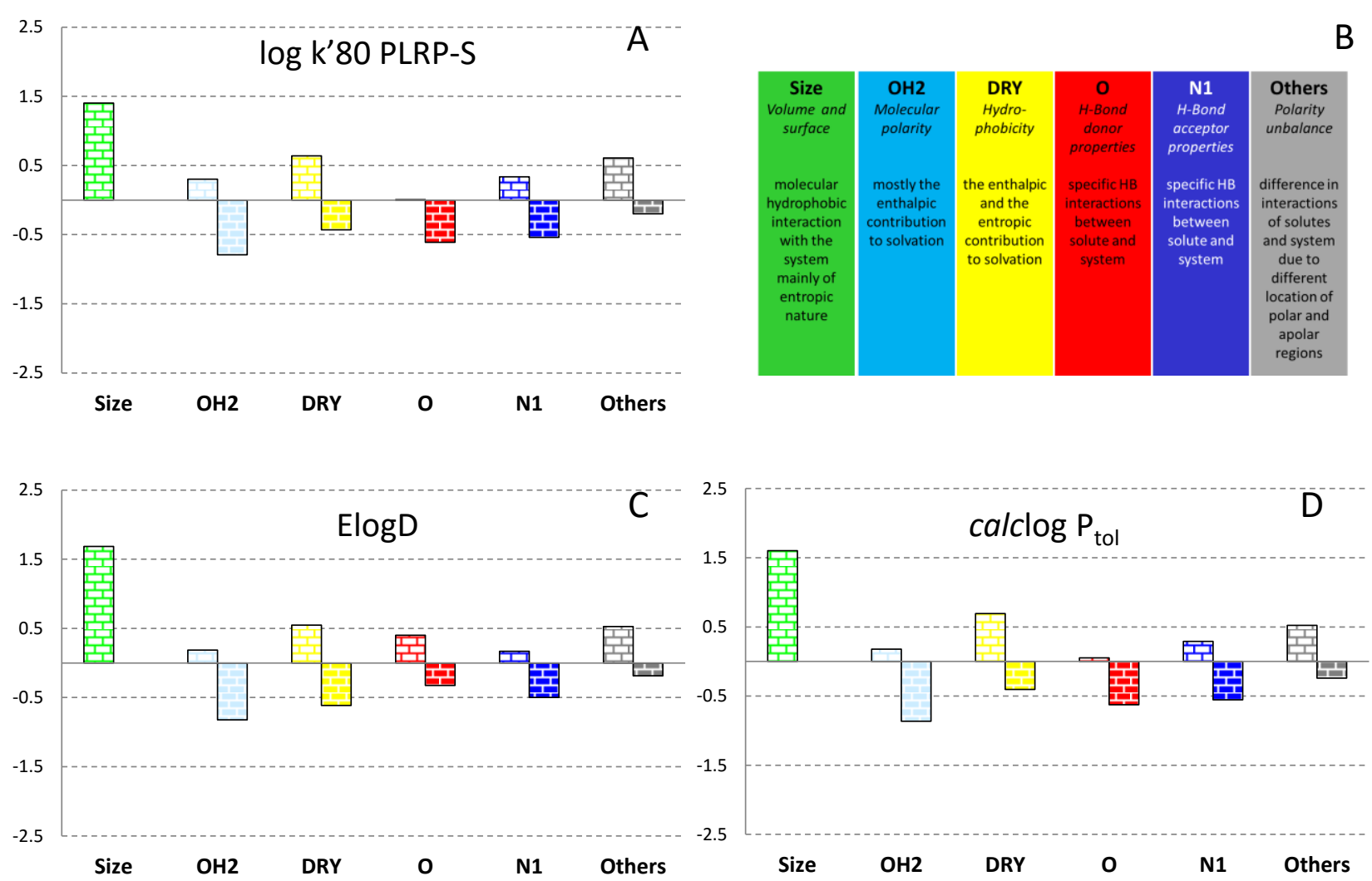

\section{Relationship with other systems}

ElogD is a validated method for $\log \mathrm{D}_{\mathrm{oct}}$ measurements. ${ }^{8}$ The $\mathrm{BR}$ analysis of $\mathrm{Elog} \mathrm{D}$ data was obtained using the same procedure as described above for log k' 80 PLRP-S. The results are shown in Figure 1C and outline the major role played in ElogD by the Size block (green). Moreover, all, but the Size block, are split in positive and negative portions. In addition, the relationship between ElogD and $\log \mathrm{k}^{\prime} 80$ PLRP-S for the dataset listed in Table 1 is shown in Figure $2 \mathrm{~A}\left(\mathrm{R}^{2}=0.54\right)$.

Figure 2. Relationship between $\log k^{\prime} 80$ PLRP-s and A) ElogD and B) calclog $P_{\text {tol }}$
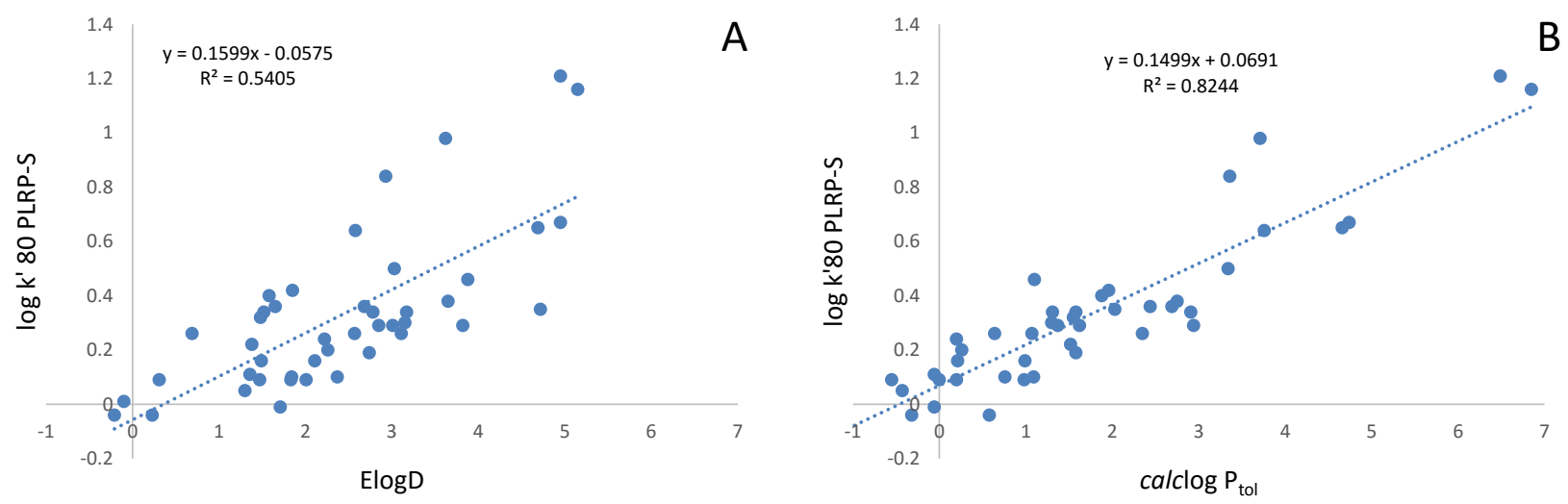
The calclog $\mathrm{P}_{\text {tol }}$ values reported in Table 1 were calculated using COSMO-RS. BR analysis output for calclog $\mathrm{P}_{\text {tol }}$ data is shown in Figure 1D, where solutes' Size (green) is the dominant block. The Hydrogen Bonds related blocks (red and blue) have mostly negative weighting. Finally, Figure $2 \mathrm{~B}$ reports the relationship between $\log$ k' 80 PLRP-S and calclog $\mathrm{P}_{\text {tol }}$ and $\mathrm{R}^{2}$ is found to be 0.82 .

\section{$\log k^{\prime} 80$ PLRP-S and IMHBs of the neutral species}

$\log$ k'80 PLRP-S for the selected pairs of compounds (the second dataset, see Experimental Part) are reported in Figure 3. Results show that all compounds with propensity to form IMHBs (denoted as S, samples), are more retained than compounds with no propensity to form IMHB (denoted as C, controls). The ${ }^{1} \mathrm{H}$ NMR chemical shifts of the pairs in DMSO- $d_{6}$ are also presented here for validation purposes. In particular, the ${ }^{1} \mathrm{H}$ chemical shifts of the proton potentially involved in the formation of IMHBs were monitored (data in Figure 3). ${ }^{5,16}$ A downfield shift was always observed in the samples containing IMHBs compared to the controls. For instance, the amide proton of c10 linked to the aromatic ring shows a downfield shift compared to the corresponding proton in $\mathbf{C D}$ (10.51 and 8.25, respectively).

The ${ }^{1} \mathrm{H}$ NMR chemical shifts in $\mathrm{CDCl}_{3}$ were also obtained on some pairs (Table S1 in Supplemental Info), however, due to solubility issues in $\mathrm{CDCl}_{3}$, only values measured in DMSO- $d_{6}$ are available for all studied compounds. The DMSO- $d_{6}$ dielectric constant is approximately 47 , and it is much more polar compared to chloroform, or to the cell membranes environment. The formation of IMHB in DMSO- $d_{6}$ requires stronger interactions compared to a less polar environment. The PLRP-S system is different in polarity compared to DMSO- $d_{6}$ or $\mathrm{CDCl}_{3}$, however, the results demonstrate that IMHB observed in DMSO- $d_{6}$ and $\mathrm{CDCl}_{3}$ (where available) are also observed in the PLRP-S system.

Figure 3. Pairs $(C=$ control, $S=$ sample $)$ selected to show the application of log k'80 PLRP-S to detect the presence of intramolecular hydrogen bonds. ${ }^{l} H$ NMR chemical shifts of the proton potentially involved in the formation of IMHBs are shown for validation purposes. Compounds lettering and numbering reflect the original assignments made in the previous studies. ${ }^{5,16}$ 


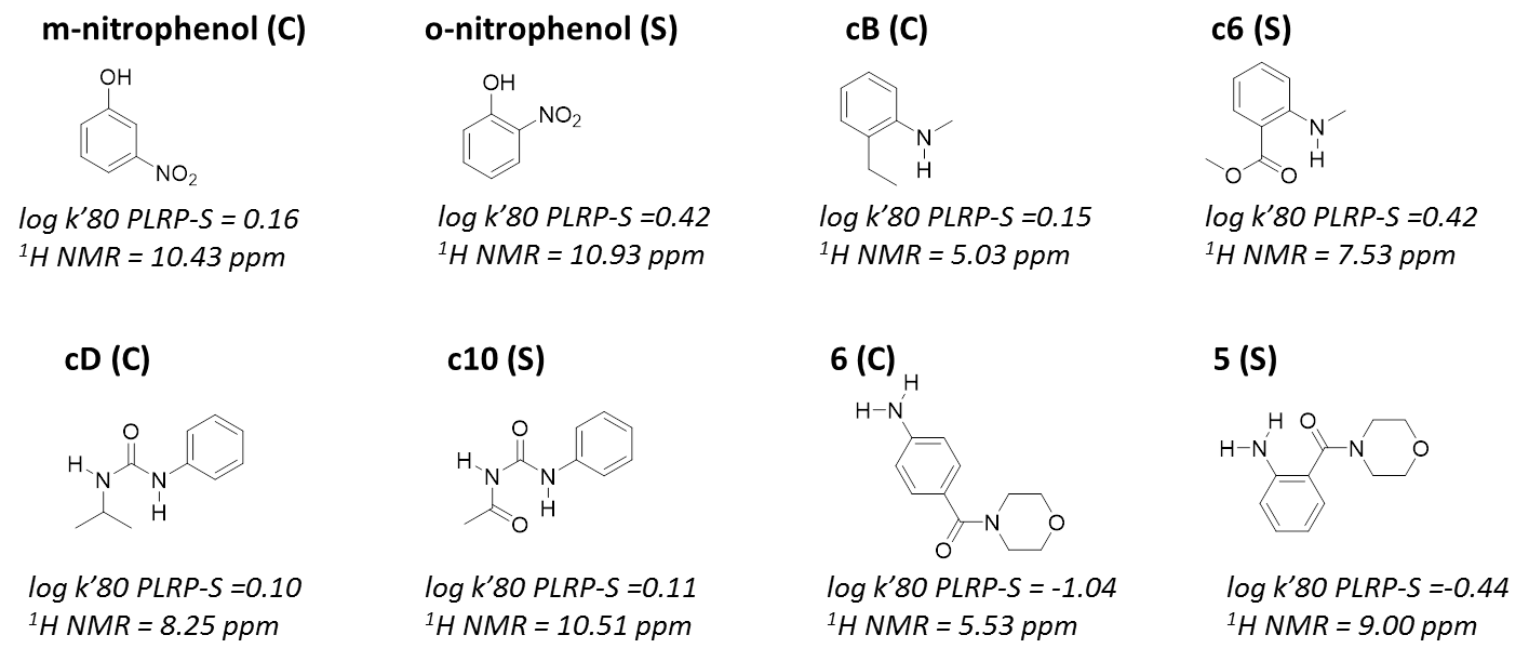

4 (C)

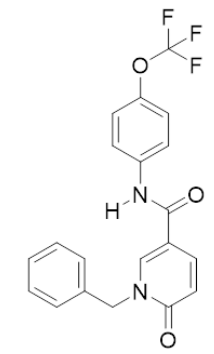

$\log k^{\prime} 80$ PLRP-S $=-0.09$

${ }^{1} \mathrm{HNMR}=10.18 \mathrm{ppm}$
3 (S)

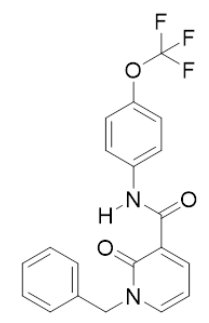

$\log k^{\prime} 80$ PLRP-S $=0.32$

${ }^{1} \mathrm{H} N \mathrm{NMR}=12.15 \mathrm{ppm}$

\section{The ionized species interactions in the PLRP-S system}

Since most drugs are partly or fully ionized at physiological pHs, we extended the study to include 76 drugs in various states of ionization and measured log k'80 PLRP-S at neutral, acidic and basic pHs. The pH measured in water-organic mixture produced apparent ${ }_{\mathrm{w}}^{\mathrm{s}} \mathrm{pH}$ values. These values are not adjusted for the presence of the organic co-solvent and are different from $\mathrm{pH}$ measured in the aqueous portions of the buffer $(\mathrm{pH}$ values measured in aqueous and co-solvent mixtures are given in Table $\mathrm{S} 2$ in Supplemental Information). The apparent ${ }_{\mathrm{w}}^{\mathrm{s}} \mathrm{pH}$ values are used throughout here, since the mobile phases were prepared with the same amount of acetonitrile, $80 \%$. In addition, the gradient was avoided, as not to change the amount of organic modifier, which could affect the ionization of the compounds and all studies were performed at isocratic chromatographic conditions.

The data are shown in Table 3. The compounds are distinguished in four groups on the basis of their ionization state at $\mathrm{pH} 7$, estimated according to MoKa's $\mathrm{pK}_{\mathrm{a}}$ predictions: neutral drugs (= predominantly neutral at $\mathrm{pH} 7$ ), acids (=predominantly negatively charged at $\mathrm{pH} 7$ ), bases (=predominantly positively charged at $\mathrm{pH} 7$ ) and three compounds (cetirizine, levodopa and methotrexate) with more complex ionization profiles.

Table 3. $\log k^{\prime} 80$ PLRP-S at different $p H$ conditions (acidic, neutral and basic) for the series of 76 compounds. The dominant species (by MoKa calculations) at specified $\mathrm{pH}$ are also reported. 


\begin{tabular}{|c|c|c|c|c|c|c|c|}
\hline & Compound & $\begin{array}{l}\log k^{\prime} 80 \\
\text { PLRP-S } \\
\left({ }^{\mathrm{s}} \mathrm{w} \text { wH 3) }\right. \\
\end{array}$ & $\begin{array}{l}\text { log k'80 } \\
\text { PLRP-S } \\
\left({ }^{\mathrm{s}} \mathrm{wpH} 7.7\right)\end{array}$ & $\begin{array}{l}\log k^{\prime} 80 \\
\text { PLRP-S } \\
\text { (s }{ }_{\text {wpH } 11)}\end{array}$ & $\begin{array}{l}\text { Ionization at } \\
\mathrm{pH}=2\end{array}$ & $\begin{array}{l}\text { Ionization } \\
\text { at pH } 7\end{array}$ & $\begin{array}{l}\text { Ionization at } \\
\mathrm{pH}=11\end{array}$ \\
\hline \multirow{19}{*}{$\begin{array}{l}\frac{n}{\pi} \\
\bar{\Xi} \\
\bar{E}\end{array}$} & Alfentanil & -0.17 & 0.22 & 0.24 & +1 & 0 & 0 \\
\hline & Antipyrine & -0.04 & -0.04 & -0.01 & 0 & 0 & 0 \\
\hline & Caffeine & -0.05 & -0.04 & -0.02 & 0 & 0 & 0 \\
\hline & Carbamazepine & 0.10 & 0.09 & 0.11 & 0 & 0 & 0 \\
\hline & Cimetidine & -0.94 & -0.16 & -0.14 & +1 & 0 & 0 \\
\hline & Colchicine & -0.07 & -0.08 & -0.05 & 0 & 0 & 0 \\
\hline & Corticosterone & 0.13 & 0.11 & 0.15 & 0 & 0 & 0 \\
\hline & Cyclosporin A & 0.31 & 0.29 & 0.31 & 0 & 0 & 0 \\
\hline & Diazepam & 0.48 & 0.47 & 0.50 & 0 & 0 & 0 \\
\hline & Etoposide & -0.10 & -0.09 & -1.26 & 0 & 0 & 0 \\
\hline & Hydrocortisone & -0.03 & -0.01 & 0.01 & 0 & 0 & 0 \\
\hline & Indinavir & -1.18 & -0.01 & 0.01 & +2 & $0 /+1 * *$ & -1 \\
\hline & Paclitaxel & 0.15 & 0.12 & 0.16 & 0 & 0 & 0 \\
\hline & Phenytoin & 0.02 & 0.01 & -0.75 & 0 & 0 & -1 \\
\hline & Ritonavir & 0.15 & 0.14 & 0.15 & +2 & 0 & 0 \\
\hline & Testosterone & 0.31 & 0.30 & 0.32 & 0 & 0 & 0 \\
\hline & Theophylline & -0.13 & -0.12 & -0.81 & $0 /+1$ & 0 & -1 \\
\hline & Trazodone & -1.06 & 0.45 & 0.47 & +1 & $0 /+1 * *$ & 0 \\
\hline & Zidovudine & -0.12 & -0.11 & -0.25 & 0 & 0 & -1 \\
\hline \multirow{15}{*}{$\frac{0}{e}$} & Atorvastatin & 0.10 & -0.12 & -1.03 & 0 & -1 & -1 \\
\hline & Entacapone & 0.05 & -0.15 & -0.64 & 0 & -1 & -2 \\
\hline & Flurbiprofen & 0.29 & -0.10 & -1.10 & 0 & -1 & -1 \\
\hline & Furosemide & -0.03 & -0.21 & -1.14 & 0 & -1 & -2 \\
\hline & Ibuprofen & 0.27 & -0.03 & -1.08 & 0 & -1 & -1 \\
\hline & Indomethacin & 0.37 & -0.06 & -1.08 & 0 & -1 & -1 \\
\hline & Naproxen & 0.21 & -0.09 & -1.10 & 0 & -1 & -1 \\
\hline & Nitecapone & 0.01 & -0.19 & -0.66 & 0 & -1 & -2 \\
\hline & Phenylbutazone & 0.10 & -0.08 & -1.05 & 0 & -1 & -1 \\
\hline & Probenecid & 0.13 & -0.20 & -1.08 & 0 & -1 & -1 \\
\hline & Salicylic acid & 0.14 & -0.22 & -1.10 & 0 & -1 & -1 \\
\hline & Tolbutamide & 0.10 & -0.17 & -0.97 & 0 & -1 & -1 \\
\hline & Tolcapone & 0.25 & -0.12 & -0.61 & 0 & -1 & -2 \\
\hline & Valsartan & 0.06 & * & $*$ & 0 & -2 & -2 \\
\hline & Warfarin & 0.28 & -0.12 & -1.03 & 0 & -1 & -1 \\
\hline \multirow{12}{*}{ 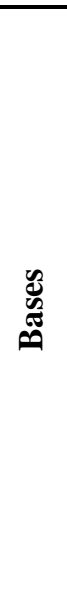 } & Amitriptyline & -0.73 & 0.58 & 0.95 & +1 & +1 & 0 \\
\hline & Amoxapine & -0.87 & 0.40 & 0.48 & +2 & +1 & 0 \\
\hline & Astemizole & $*$ & 0.41 & 0.39 & +2 & +1 & 0 \\
\hline & Buspirone & -0.98 & 0.42 & 0.44 & +2 & +1 & 0 \\
\hline & Chlorpromazine & -0.79 & 0.91 & 1.26 & +2 & +1 & 0 \\
\hline & Citalopram & -1.01 & 0.17 & 0.44 & +1 & +1 & 0 \\
\hline & Clozapine & -1.01 & 0.45 & 0.47 & +3 & +1 & 0 \\
\hline & Codeine & -1.06 & 0.02 & 0.05 & +1 & +1 & 0 \\
\hline & Diltiazem & -0.93 & 0.31 & 0.37 & +1 & +1 & 0 \\
\hline & Diphenhydramine & -0.93 & 0.24 & 0.53 & +1 & +1 & 0 \\
\hline & Domperidone & -0.97 & 0.00 & 0.03 & +1 & +1 & -1 \\
\hline & Fentanyl & -0.89 & 0.49 & 0.60 & +1 & +1 & 0 \\
\hline
\end{tabular}




\begin{tabular}{|c|c|c|c|c|c|c|c|}
\hline & Fluoxetine & -0.97 & 0.18 & 0.52 & +1 & +1 & 0 \\
\hline & Galanthamine & -1.08 & 0.02 & 0.09 & +1 & +1 & 0 \\
\hline & Haloperidol & -0.97 & 0.38 & 0.46 & +1 & +1 & 0 \\
\hline & Imatinib & -1.12 & 0.10 & 0.08 & +4 & +1 & 0 \\
\hline & Lidocaine & -1.00 & 0.28 & 0.32 & +1 & +1 & 0 \\
\hline & Maprotiline & -0.71 & 0.26 & 0.84 & +1 & +1 & 0 \\
\hline & Mesoridazine & -1.01 & 0.25 & 0.47 & +2 & +1 & 0 \\
\hline & Methadone & -0.90 & 0.23 & 0.93 & +1 & +1 & 0 \\
\hline & Metoclopramide & -1.10 & -0.04 & 0.14 & +2 & +2 & 0 \\
\hline & Mirtazapine & -0.49 & 0.36 & 0.36 & +2 & +1 & 0 \\
\hline & Morphine & -1.00 & -0.08 & -0.08 & +1 & +1 & -1 \\
\hline & Oxycodone & -1.15 & 0.21 & 0.35 & +1 & +1 & 0 \\
\hline & Perphenazine & -0.81 & 0.66 & 0.67 & +2 & +1 & 0 \\
\hline & Propranolol & -1.03 & 0.30 & 0.44 & +1 & +1 & 0 \\
\hline & Quinidine & -1.13 & 0.40 & 0.23 & +2 & +1 & 0 \\
\hline & Quinine & -1.06 & 0.39 & 0.20 & +2 & +1 & 0 \\
\hline & Risperidone & -1.10 & 0.17 & 0.19 & +2 & +1 & 0 \\
\hline & Saquinavir & -0.97 & 0.17 & 0.20 & +1 & +1 & 0 \\
\hline & Sertraline & -0.77 & 0.75 & 1.09 & +1 & +1 & 0 \\
\hline & Sildenafil & -1.03 & 0.20 & -0.12 & +2 & +1 & -1 \\
\hline & Sumatriptan & -1.18 & -0.10 & -0.02 & +1 & +1 & -1 \\
\hline & Terfenadine & -0.81 & 0.43 & 0.84 & +1 & +1 & 0 \\
\hline & Thioridazine & -0.72 & 0.96 & 1.37 & +2 & +1 & 0 \\
\hline & Venlafaxine & -1.03 & 0.16 & 0.60 & +1 & +1 & 0 \\
\hline & Verapamil & -1.00 & 0.33 & 0.52 & +1 & +1 & -1 \\
\hline & Cetirizine & -0.66 & -0.09 & -1.02 & +2 & $+1 ;-1 * * *$ & -1 \\
\hline 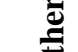 & Levodopa & -0.41 & -0.10 & -1.26 & +1 & $+1 ;-1 * * *$ & -2 \\
\hline$\overline{0}$ & Methotrexate & -1.08 & 0.15 & -2.46 & +2 & -2 & -2 \\
\hline
\end{tabular}

*used to measure dead time, $t_{0}$ (see the Experimental Section)

**both species present at about $50 \%$

***zwitterions

The log k'80 PLRP-S values listed in Table 3 are plotted $v s \mathrm{pH}$ in Figure 4 to help with interpretation of the results.

Figure 4. The variation of log k'80 PLRP-S vs pH for drugs listed in Table 3: A) acids, B) bases and C) neutrals. The connecting lines are colored and enlarged for compounds highligted in the discussion. 

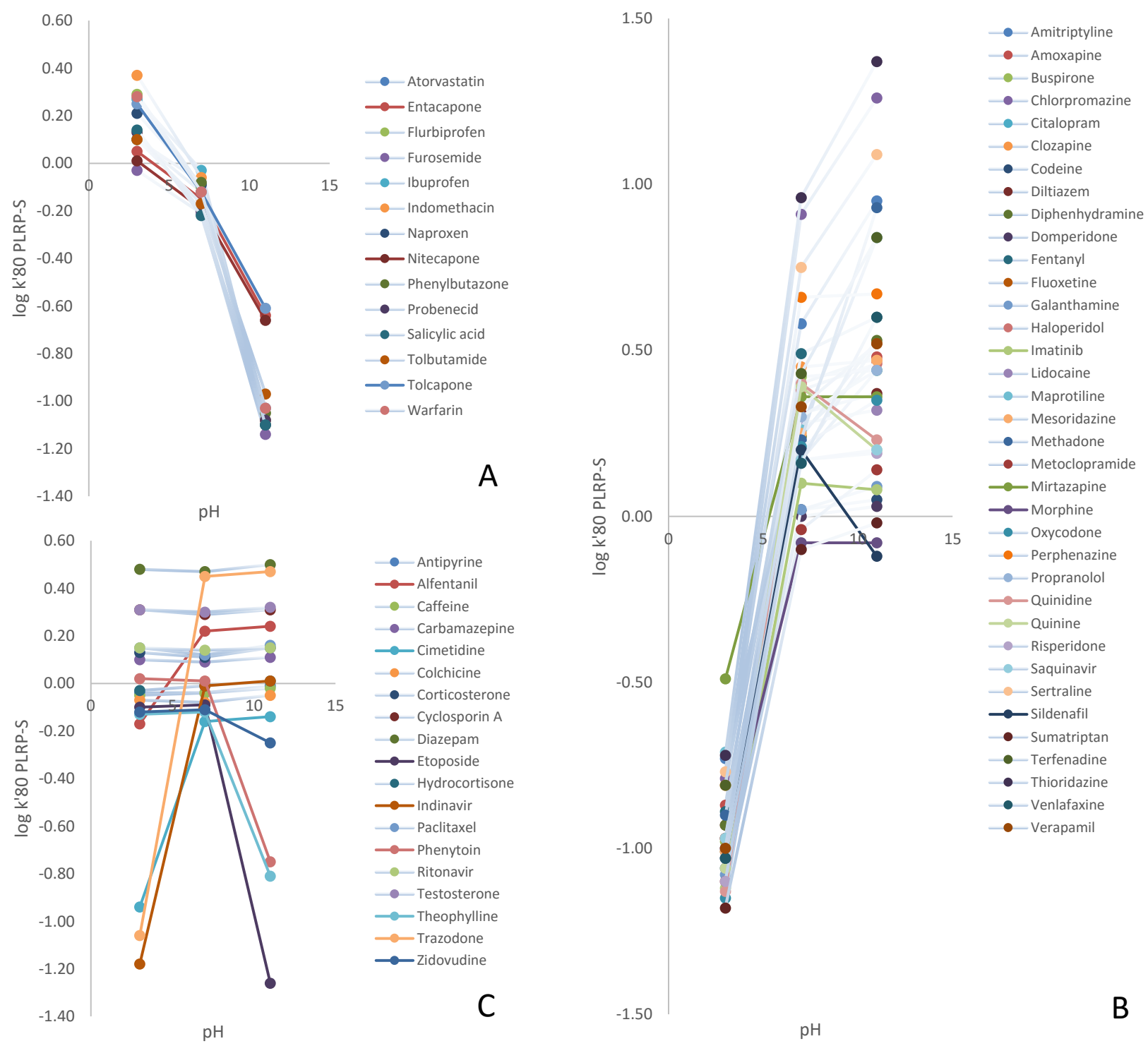

\section{Acids (Figure 4A)}

All investigated acids show a drop in log k'80 PLRP-S when the mobile phase $\mathrm{pH}$ moves from acidic to basic $\mathrm{pHs}$. At the basic $\mathrm{pH}$, where these molecules are predominantly in their anionic form, their log k' 80 PLRP-S is rather constant and close to -1.0. Entacapone, nitecapone and tolcapone show a peculiar behavior since their log k'80 PLRP-S value is about -0.6 , significantly higher than those registered for other acids. Capones have two acidic centers $\left(\mathrm{pK}_{\mathrm{a}} \mathrm{s} \text { about } 5 \text { and } 9\right)^{22}$ and thus their dianionic forms are predominant at basic pHs.

\section{Bases (Figure 4B)}

Generally speaking, for most monobases the trend in Figure 4B is opposite to monoacids, i.e. the drop in log k'80 PLRP-S is found when passing from basic to acidic pHs. However, at the acidic $\mathrm{pH}$, when monobases are expected to be mostly in their cationic form, log k'80 PLRP-S values vary for all drugs. Mirtazapine, which is expected to be diprotonated at acidic pH (Table 3), shows the higher value of log k' 80 PLRP-S. 
Mirtazapine, imatinib and morphine do not show any decrease in log k'80 PLRP-S when passing from basic to neutral $\mathrm{pH}$. Mirtazapine and imatinib are expected to protonate when passing from basic to neutral $\mathrm{pH}$. Conversely, morphine is expected to change its ionization state from -1 to +1 during this passage.

Quinine, quinidine and sildenafil show an increase in log k'80 PLRP-S when passing from basic to neutral $\mathrm{pH}$. For quinine and quinidine this corresponds to a passage from the neutral to the cationic species. For sildenafil the passage from basic to neutral conditions corresponds to a change in its protonation state from 1 to +1 .

\section{Neutrals (Figure 4C)}

For many neutral drugs log k'80 PLRP-S values were rather constant at any pH. Four drugs (alfentanil, cimetidine, trazodone and indinavir) show a drop in log k'80 PLRP-S at acidic pHs. MoKa predicts that at acidic pHs the four drugs are protonated. Ritonavir is also predicted diprotonated but log k'80 PLRP-S does not vary with the $\mathrm{pH}$. Zidovudine, phenytoin, theophylline and etoposide show a drop in log k'80 PLRP-S at basic pHs. All but etoposide are expected to be negatively charged at basic $\mathrm{pH}$. Indinavir has an opposite behavior compared to etoposide, since it is expected to be negatively charged at basic $\mathrm{pH}$, but its log k' 80 PLRP-S does not vary with the $\mathrm{pH}$.

\section{Other molecules}

The two zwitterions (cetirizine and levodopa) have consistent behavior. Cetirizine shows the largest log k' 80 PLRP-S when the zwitterion is the dominant species as shown in the literature. ${ }^{23}$ Levodopa shows a similar trend to cetirizine: the cation is largely more lipophilic than the anion, whereas the zwitterion is the most lipophilic species. Methotrexate has a more complex ionization profile and the zwitterionic species is barely present. ${ }^{24}$

\section{Discussion}

\section{PLRP-S system set-up}

The choice of the column was made from the analysis of literature data. We evaluated the balance of the intermolecular interactions governing chromatographic retention in HPLC systems with polymeric columns described in the literature ${ }^{10}$ (data not shown) through the BR analysis (see below for details). ${ }^{14}$ We found that the retention on polymeric column is governed by the size of the solutes and, also, by the interactions between the hydrogen bond donor (HBD) properties of the solutes and the hydrogen bond acceptor (HBA) properties of the system. This is in line with the analysis recently reported in the literature for $\log \mathrm{P}_{\text {tol }},{ }^{17}$ which is considered a relevant system for describing nonpolar environments.

The mobile phase composition was chosen as a result of a number of considerations. Acetonitrile (ACN) was preferred for its aprotic nature, better interaction with the stationary phase and improved elution, resulting in better peak shape, compared to methanol-containing mobile phase, which demonstrated significant peak tailing. The composition of the mobile phase ( $80 \%$ ACN: $20 \%$ buffer) was selected to achieve the best compromise between speed and selectivity. The isocratic conditions were preferred over the gradient for a number of reasons. Firstly, we recently showed the influence of the changes in the mobile phase composition 
in a gradient, on the balance of forces governing retention in a given system ${ }^{14}$ and we wanted to avoid such variability. Then we verified that $80 \% \mathrm{ACN}$ ensured rapid elution, while still providing adequate separation. Finally, isocratic conditions were preferred for the application of a Quantitative Structure-Properties Relationships (QSPR) approach to characterize the system.

\section{$\log$ k'80 PLRP-S characterization and its relationship with calclog $\mathbf{P}_{\text {tol }}$ and ElogD}

Log k'80 PLRP-S was characterized using BR analysis output plots (Figure 1A). This tool provides information about the balance of the intermolecular interactions governing the system. In a BR plot, blocks with positive weighting (e.g. the green block in Figure 1A) show how much the property described by the block increases retention, whereas blocks with negative weighting (e.g., the red block) indicate how much the property decreases log k'80 PLRP-S values. The positive value of the Size block (green) in Figure 1A supports that larger compounds have longer retention under investigated PLRP-S conditions. This evidence is shared by all chromatographic RP systems. ${ }^{19}$ The Size and HBD solutes' properties (O block, red) are almost entirely attributed either in positive or negative portions. That shows the modest noise and intercorrelation of these descriptors and thus confirms their relevance to the model. In particular, the relevance of the red block supports the applicability of log k' 80 PLRP-S to discriminate the propensity of candidates to form IMHBs, as described in detail below.

For comparative purposes, we obtained ElogD and calclog $\mathrm{P}_{\text {tol }}$ for the same 44 neutral compounds chosen for characterizing the PLRP-S system. ElogD was selected since it is a chromatographic method widely appreciated for determining $\log \mathrm{D}_{7.4}$ in the standard system, i.e. octanol/water. The presence of a relationship between $\log \mathrm{k}^{\prime} 80 \mathrm{PLRP}-\mathrm{S}$ and $\mathrm{E} \log \mathrm{D}\left(\mathrm{R}^{2}=0.43\right)$ (Figure $\left.2 \mathrm{~A}\right)$ is mostly due to the solutes dimensions: the larger the compounds the easier they stay in the nonpolar phase. That is verified by BR analysis which shows a similar trend for the Size block present in both descriptors (Figure 1A and 1C).

The $\log \mathrm{P}_{\text {tol }}$ was selected as a second reference descriptor to compare log k'80 PLRP-S data with values obtained in nonpolar system. Measured $\log \mathrm{P}_{\text {tol }}$ values are not available for many compounds in the dataset due to difficulties of measurements, as described in the literature ${ }^{5}$ and we used calculated values for this reason. The linear regression in Figure $2 \mathrm{~B}$ shows a slope significantly different from 1. However, BR analysis demonstrated that log k'80 PLRP-S and calclog $\mathrm{P}_{\text {tol }}$ (Figure1A and 1D) are governed by the similar balance of intermolecular forces between the solutes and the system.

\section{Identification of IMHB of neutral compounds using log k'80 PLRP-S}

The identification of compounds likely to form IMHBs is an important drug design consideration since it is related to increased membrane permeability. ${ }^{18}$

NMR chemical shift is the most widely known tool $^{25}$ for IMHB verification, albeit, it is often limited by the solubility issues. ${ }^{5} \Delta \log \mathrm{P}_{\text {oct-tol }}$ and EPSA techniques have recently gained relevance. However, the existing methods do not cover the variability of the physiological environments and log k'80 PLRP-S could serve as an additional tool.

Given the relevance of the solutes HBD properties (red block) in Figure 1A, the PLRP-S system is expected to detect the presence of IMHBs. Indeed, the formation of IMHB "hides" the HB donor and HB acceptor of a 
solute and that could be detected by the PLRP-S system. Similar to $\Delta \log \mathrm{P}_{\text {oct-tol }}$ and EPSA methods, the $\log$ k'80 PLRP-S has to be used in a pair wise way to limit the interference of Size. That is clearly shown by the BR analysis above. Indeed, for a pair of close analogs we can assume that both control and sample have similar size and hydrophobic properties and thus their difference in log k'80 PLRP-S is mostly due to the difference in HBD properties.

The experimental results for the selected pairs (data in Figure 3) showed that log k'80 PLRP-S values are differentiating the propensity of the investigated neutral compounds to form IMHBs. This is exemplified by the comparison of the $\mathbf{c B}$ (the control) and $\mathbf{c 6}$ (the sample) pair.

A $\log$ k'80 PLRP-S of 0.15 was measured for $\mathbf{c B}$. In the absence of IMHB $\mathbf{c 6}$ is expected to be more polar and thus less retained than $\mathbf{c B}$. Since the $\log$ k' 80 PLRP-S of $\mathbf{c 6}$ is 0.42 , it supports the presence of IMHBs.

The ${ }^{1} \mathrm{H}$ NMR chemical shift analysis also provides evidence on the propensity of samples to form IMHBs. Interestingly, both log k'80 PLRP-S results and ${ }^{1} \mathrm{H}$ NMR chemical shifts support formation of IMHB in all examined samples. Although, the two methods provide the information in different ways. For instance c10 has modest propensity to form IMHBs according to log k'80 PLRP-S (little difference with its control, cD), but high propensity according to NMR data (large difference in the chemical shift). These variations could be ascribed to the differences in polarity of the two systems and in the balance of factors governing chromatographic retention and chemical shift. $\Delta \log \mathrm{P}_{\text {oct-tol data }}$ analysis ${ }^{5}$ (as described in the Supporting Information, Table S2) also supports formation of IMHB in these compounds.

Summing up, log k'80 PLRP-S can be combined with known tools such as $\Delta \log \mathrm{P}_{\text {oct-tol }}{ }^{5}$ and EPSA ${ }^{16}$ and also with ${ }^{1} \mathrm{H}$ NMR data, to produce a reliable picture of how different physiological conditions modulate the propensity of neutral drug candidates to form IMHBs.

\section{Log k'80 PLRP-S and solutes ionization profiling across pHs}

The stability of the PLRP-S polymeric column under different $\mathrm{pH}$ conditions is an important feature of this type of stationary phase. That was already verified in a study which reported the retention factors of some ionizable compounds. ${ }^{26} \mathrm{We}$ decided to explore the advantages of PLRP-S column stability for characterizing ionizable compounds.

\section{The "charge-flush" concept}

Figure 4 shows that PLRP-S system is very sensitive to the solutes ionization. Generally speaking, log k' 80 PLRP-S decreases when passing from $\mathrm{pHs}$ in which the neutral species is dominant to $\mathrm{pHs}$ in which the ionized species are mostly present. This finding gives the basis to define the "charge-flush" concept illustrated in Figure 4. In practice, the "charge-flush" concept highlights that under the studied PLRP-S conditions the fully charged compounds are poorly retained, hence "flushed".

Another observation evident from Figure 4 is different sensitivity of the PLRP-S system for positively and negatively charged compounds. In particular, in PLRP-S system, the monocations are generally more retained than monoanions.

These findings could be used to obtain two kinds of information: a) verify predicted $\mathrm{pK}_{\mathrm{a}}$; b) estimate the propensity of monoanions to form IMHBs. 


\section{Verification of $\mathrm{pK}_{\mathrm{a}}$ prediction}

In our experience the $\mathrm{pK}_{\mathrm{a}}$ prediction accuracy is often inadequate, especially, when more than one ionization center is present. The graphic representation reported in Figure 4 could help with analysis of experimental data in relation to ionization state across $\mathrm{pH}$ ranges. It should be noted that while $\mathrm{MoKa}_{\mathrm{pK}}$ calculations are done in aqueous conditions, the logk' 80 PLRP-S is determined in the presence of significant amount of acetonitrile in the mobile phase. MoKa and other $\mathrm{pK}_{\mathrm{a}}$ models are routinely used by medicinal chemists to estimate ionization of molecules, including in biological fluids and other physiological environments. Therefore, we considered it would be practical to relate the observed interactions to the aqueous ionization scale routinely used by the drug designers.

Let's take indinavir as an example from the neutral group. An acidic $\mathrm{pK}_{\mathrm{a}}$ of about 10.5 is predicted by MoKa. This is surprising since it is associated with an amide group. The experimental results show no decrease in log k'80 PLRP-S when passing from neutral to basic $\mathrm{pH}$, which confirms that an acidic $\mathrm{pK}_{\mathrm{a}}$, if present, has a higher value than the predicted 10.5. In another example, etoposide is predicted to be neutral along the entire pH scale by MoKa. However, the low log k'80 PLRP-S value registered at basic pH suggests that the phenolic $\mathrm{OH}$ is probably more acidic than expected. For ritonavir MoKa predicts two basic $\mathrm{pK}_{\mathrm{a}} \mathrm{s}$ (2.45 and 3.85), attributed to two thiazole nitrogens. Log k'80PLRP-S data confirm that the nitrogens are, probably, less basic, since measured values at $\mathrm{pH} 7$ and $\mathrm{pH} 2$ are very close ( 0.15 and 0.14 , respectively), whereas a drop in log k'80 PLRP-S is expected in the presence of an additional ionized center.

In a group of bases morphine also shows a strange behavior. At both neutral and acidic $\mathrm{pHs}$ it is expected to be monoprotonated, but its log k'80 PLRP-S is considerably higher at acidic pH (-0.08 vs -1.00 , respectively). These experimental data could be justified by the presence of a zwitterionic species ${ }^{27}$ not evidenced by MoKa calculations. Imatinib shows the same log k'80 PLRP-S at basic and neutral pHs, but this is not consistent with the predicted $\mathrm{pK}_{\mathrm{a}}$ values, which indicates that at $\mathrm{pH}=7$, the cation is the dominant species. In other words, the basic nitrogen $\left(\mathrm{pK}_{\mathrm{a}} 8.46\right)$ is less basic than predicted. Finally, mirtazapine seems to share the same behavior as imatinib.

It could be argued that these differences are due to the high content of acetonitrile in the mobile phase in the system. That, of course, has an impact; however it could only justify a decrease in the acid/base strength and not an increase, as registered for etoposide..$^{29,30}$

These results demonstrated that log k'80 PLRP-S is very sensitive to ionization and could be used to determine the presence of ionization centers and their nature over a wide range of $\mathrm{pH}$ (1 to 12), for monitoring the ionization states of series of compounds in early stage of drug discovery when $\mathrm{pK}_{\mathrm{a}}$ are not yet measured.

\section{IMHB of ionized species detection by log $k^{9} 80$ PLRP-S}

Insights into the propensity of monoanions to form IMHBs could be obtained from the PLRP-S system. This is evident when log k'80 PLRP-S data of the three COMT inhibitors (entacapone, nitecapone and tolcapone) are analyzed (Figure 4A). The monoanionic forms are mostly present at neutral $\mathrm{pH}$ and have the propensity to form IMHBs as shown in Figure 5 for tolcapone as an example ${ }^{22}$. Their log k'80 PLRP-S is constant and 
about 0.1 . This value is considerably higher than values registered for all other anions (about -1.0), which do not have the propensity to form IMHBs. Here log k'80 PLRP-S provides information on the propensity of deprotonated acidic compounds to form IMHBs in nonpolar environments.

Figure 5. Tolcapone $\left(p K_{a} s=4.64,10.20\right)$ chemical structure in neutral form and IMHB in the monoanionic form $^{22}$

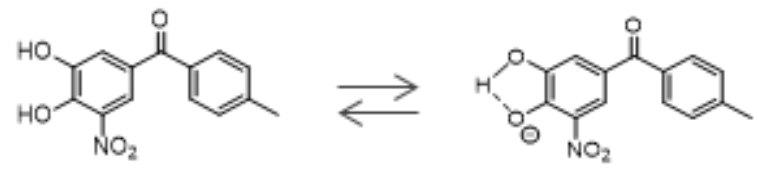

\section{Conclusion}

This study describes a fast chromatographic method to obtain a new physico-chemical index (log k' 80 PLRP-S) defining the lipophilicity of compounds in nonpolar environment such as the interior of a membrane.

Since the propensity to form IMHBs is strongly dependent on the environment, this specific descriptor is expected to help highlight the propensity of neutral compounds to form IMHBs in different matrixes. The proposed log k'80 PLRP-S descriptor could be used in combination with existing tools $\left(\Delta \log \mathrm{P}_{\text {oct-tol }}\right.$ and EPSA) in drug discovery projects.

Moreover, log k'80 PLRP-S is the only descriptor available today that provides information on the propensity of deprotonated acidic compounds to form IMHBs in nonpolar environments.

Since log k'80 PLRP-S is very sensitive to ionization it could be used to determine the presence of ionization centers and their nature in nonpolar environments. The PLRP-S column stability over a wide range of pH (1 to 12) allows for log k' 80 PLRP-S to be used for monitoring the ionization states of series of compounds in early stage of drug discovery when $\mathrm{pK}_{\mathrm{a}}$ are not yet measured.

Summing up, log k'80 PLRP-S provides unique information about the properties of drug candidates in a variety of different environments and warrants being integrated in drug discovery projects.

\section{Acknowledgement}

The authors thank Dr. Michael J. Shapiro for the critical reading of the manuscript. 


\section{References}

(1) Waring, M. J. Lipophilicity in Drug Discovery. Expert Opin. Drug Discov. 2010, 5, 235-248.

(2) Gramse, G.; Dols-Perez, A.; Edwards, M. A.; Fumagalli, L.; Gomila, G. Nanoscale Measurement of the Dielectric Constant of Supported Lipid Bilayers in Aqueous Solutions with Electrostatic Force Microscopy. Biophys. J. 2013, 104, 1257-1262.

(3) Li, L.; Li, C.; Zhang, Z.; Alexov, E. On the Dielectric "Constant" of Proteins: Smooth Dielectric Function for Macromolecular Modeling and Its Implementation in DelPhi. J. Chem. Theory Comput. 2013, 9, 2126-2136.

(4) Natesan, S.; Wang, Z.; Lukacova, V.; Peng, M.; Subramaniam, R.; Lynch, S.; Balaz, S. Structural Determinants of Drug Partitioning in N - Hexadecane/Water System. J.Chem.Inf.Model. 2013, 53(6),1424-1435.

(5) Shalaeva, M.; Caron, G.; Abramov, Y. A.; Connell, T. N. O.; Plummer, M. S.; Yalamanchi, G.; Farley, K. A.; Goetz, G. H.; Philippe, L.; Shapiro, M. J. Integrating Intramolecular Hydrogen Bonding ( IMHB ) Considerations in Drug Discovery Using $\Delta \log P$ As a Tool. J. Med. Chem. 2013, $56,4870-4879$.

(6) Poole, S. K.; Poole, C. F. Separation Methods for Estimating Octanol-water Partition Coefficients. J. Chromatogr. B 2003, 797, 3-19.

(7) Valkó, K. Application of High-Performance Liquid Chromatography Based Measurements of Lipophilicity to Model Biological Distribution. J. Chromatogr. A 2004, 1037, 299-310.

(8) Lombardo, F.; Shalaeva, M. Y.; Tupper, K. A.; Gao, F. ElogD Oct: A Tool for Lipophilicity Determination in Drug Discovery. 2. Basic and Neutral Compounds. J. Med. Chem. 2001, 44, 24902497.

(9) Goetz, G. H.; Philippe, L.; Shapiro, M. J. EPSA: A Novel Supercritical Fluid Chromatography Technique Enabling the Design of Permeable Cyclic Peptides. ACS Med. Chem. Lett. 2014, 5, 1167 1172 .

(10) Abraham, M. H.; Chadha, H. S.; Leitao, R. a. .; Mitchell, R. C.; Lambert, W. J.; Kaliszan, R.; Nasal, A.; Haber, P. Determination of Solute Lipophilicity, as Log P(octanol) and Log P(alkane) Using Poly(styrene-divinylbenzene) and Immobilised Artificial Membrane Stationary Phases in ReversedPhase High-Performance Liquid Chromatography. J. Chromatogr. A 1997, 766, 35-47.

(11) Fikri, K.; Debord, J.; Bollinger, J-C; Cledat, D., Penicaut, B.; Robert, J-M. HPLC lipophilicity studies for some (hetero) arylamides derived from 2-amino 4,6 -dimethyl pyridine : introduction of an hydrogen bond descriptor. J. Liq. Chromatogr. 2011, 34, 1356-1366.

(12) Lambert, W. J.; Wright, L. A. Prediction of Alkane-Water Partition Coefficients Using a C18 Derivatized Polystirene-Divinyl Benzene Stationary Phase. J. Chromatogr. 1989, 464, 400-404.

(13) Jensen, D. A.; Gary, R. K. Estimation of Alkane-water $\log$ P for Neutral, Acidic, and Basic Compounds Using an Alkylated Polystyrene-Divinylbenzene High-Performance Liquid Chromatography Column. J. Chromatogr. A, 2015, 1417, 21-29.

(14) Caron, G.; Vallaro, M.; Ermondi, G. The Block Relevance (BR) Analysis to Aid Medicinal Chemists to Determine and Interpret Lipophilicity. MedChemComm 2013, 4, 1376-1381. 
(15) Sköld, C.; Winiwarter, S.; Wernevik, J.; Bergström, F.; Engström, L.; Allen, R.; Box, K.; Comer, J.; Mole, J.; Hallberg, A.; Lennernäs, H.; Lundstedt, T.; Ungell, A. L.; Karlén, A. Presentation of a Structurally Diverse and Commercially Available Drug Data Set for Correlation and Benchmarking Studies. J. Med. Chem. 2006, 49, 6660-6671.

(16) Goetz, G. H.; Farrell, W.; Shalaeva, M.; Sciabola, S.; Anderson, D.; Yan, J.; Philippe, L.; Shapiro, M. J. High Throughput Method for the Indirect Detection of Intramolecular Hydrogen Bonding. J. Med. Chem. 2014, 57, 2920-2929.

(17) Ermondi, G.; Visconti, A.; Esposito, R.; Caron, G. The Block Relevance (BR) Analysis Supports the Dominating Effect of Solutes Hydrogen Bond Acidity on $\Delta \log \mathrm{P}($ oct-Tol). Eur. J. Pharm. Sci. 2014, $53,50-54$.

(18) Kuhn, B.; Mohr, P.; Stahl, M. Intramolecular Hydrogen Bonding in Medicinal Chemistry. J. Med. Chem. 2010, 53, 2601-2611.

(19) Ermondi, G.; Caron, G. Molecular Interaction Fields Based Descriptors to Interpret and Compare Chromatographic Indexes. J. Chromatogr. A 2012, 1252, 84-89.

(20) Potter, T.; Ermondi, G.; Newbury, G.; Caron, G. Relating Caco-2 Permeability to Molecular Properties Using Block Relevance Analysis. Med. Chem. Commun. 2014, 6, 626-629.

(21) Caron, G.; Visentin, S.; Pontremoli, C.; Ermondi, G. Profile of the Intermolecular Forces Governing the Interaction of Drugs with Mucin. Int. J. Pharm. 2015, 488, 67-69.

(22) Novaroli, L.; Bouchard, G.; Caron, G.; Fruttero, R.; Carrupt, P.-A.; Testa, B. The Lipophilicity Behaviour of COMT Inhibitors. Chimia (Aarau). 2002, 56, 344.

(23) Plember van Balen, G.; Caron, G.; Ermondi, G.; Pagliara, a; Grandi, T.; Bouchard, G.; Fruttero, R.; Carrupt, P. a; Testa, B. Lipophilicity Behaviour of the Zwitterionic Antihistamine Cetirizine in Phosphatidylcholine Liposomes/water Systems. Pharm. Res. 2001, 18, 694-701.

(24) Shalaeva, M.; Kenseth J, Lombardo F, Bastin A. Measurement of Dissociation Constants (pKa Values) of Organic Compounds by Multiplexed Capillary Electrophoresis Using Aqueous and Cosolvent Buffers. J.Pharm.Sci 2008, 97, 2581-2606.

(25) Abraham, M. H.; Abraham, R. J.; Acree, W. E.; Aliev, A. E.; Leo, A. J.; Whaley, W. L. An NMR Method for the Quantitative Assessment of Intramolecular Hydrogen Bonding; Application to Physicochemical, Environmental, and Biochemical Properties. J.Org. Chem. 2014, 79, 11075-11083.

(26) Ruiz, R.; Ruiz-Ángel, M. J.; García-Álvarez-Coque, M. C.; Ràfols, C.; Rosés, M.; Bosch, E. Hydrophobic and Cation Exchange Mechanisms in the Retention of Basic Compounds in a Polymeric Column. J. Chromatogr. A 2004, 1028, 139-148.

(27) Mazák, K.; Noszál, B. Lipophilicity of Morphine Microspecies and Their Contribution to the Lipophilicity Profile. Eur. J. Pharm. Sci. 2012, 45, 205-210.

(28) Gagliardi, L. G.; Castells, C. B.; Ràfols, C.; Rosés, M.; Bosch, E. 6 Conversion Parameter between $\mathrm{pH}$ Scales (w spH and s spH) in Acetonitrile/water Mixtures at Various Compositions and Temperatures. Anal. Chem. 2007, 79, 3180-3187.

(29) Espinosa, S.; Bosch, E.; Rosés, M. Retention of Ionisable Compounds in High-Performance Liquid Chromatography. 14. Acid-Base pK Values in Acetonitrile-Water Mobile Phases. J. Chromatogr. A 2002, 964, 55-66. 


\section{Supporting Information}

\section{Tables}

Table S1. Experimental data for pairs in Figure 3.

\begin{tabular}{|l|l|l|l|l|l|l|l|}
\hline $\begin{array}{l}\text { Compound } \\
\text { ID }\end{array}$ & $\log \mathbf{P}_{\text {oct }}$ & $\log \mathbf{P}_{\text {tol }}$ & $\mathbf{\Delta} \log \mathbf{P}_{\text {oct-tol }}$ & $\mathbf{I M H B}$ & $\begin{array}{l}\boldsymbol{\delta} \text { DMSO-d6, } \\
\mathbf{p p m}\end{array}$ & $\begin{array}{l}\boldsymbol{\delta} \text { CDC13, } \\
\mathbf{p p m}\end{array}$ & $\begin{array}{l}\text { ogk'80 } \\
\text { PLRP-S }\end{array}$ \\
\hline m-nitrophenol & 1.73 & 0.34 & 1.39 & control & 10.43 & 5.20 & 0.16 \\
\hline o-nitrophenol & 1.57 & 2.26 & -0.69 & sample & 10.93 & 10.60 & 0.42 \\
\hline $\mathbf{c B}^{5}$ & 1.91 & 1.96 & -0.05 & control & 5.03 & 3.68 & 0.15 \\
\hline $\mathbf{c 6}^{5}$ & $2.1 *$ & $2.1 *$ & 0 & sample & 7.53 & 7.64 & 0.42 \\
\hline $\mathbf{c D}^{5}$ & 1.76 & 0.13 & 1.63 & control & 8.25 & 6.01 & 0.10 \\
\hline $\mathbf{c 1 0}^{5}$ & 1.43 & 0.71 & 0.72 & sample & 10.51 & 10.37 & 0.11 \\
\hline $\mathbf{6}^{16}$ & -0.34 & -1.08 & 0.74 & control & 5.53 & na & -1.04 \\
\hline $\mathbf{5}^{16}$ & -0.01 & -0.29 & 0.28 & sample & 9.0 & na & -0.44 \\
\hline $\mathbf{4}^{16}$ & $4.8^{* *}$ & $3.84^{* * *}$ & 0.96 & control & 10.18 & na & -0.09 \\
\hline $\mathbf{3}^{\mathbf{1 6}}$ & $5.6 * *$ & $5.33^{* * *}$ & 0.27 & sample & 12.15 & na & 0.32 \\
\hline
\end{tabular}

* - uncertain value due to aggregation/quantification issues.

** ElogD values

***calclog $\mathrm{P}_{\text {tol }}$

When $\Delta \log \mathrm{P}_{\text {oct-tol }}$ of the control is larger than $\Delta \log \mathrm{P}_{\text {oct-tol }}$ of the sample, the sample has high propensity to form IMHB. This is verified for all pairs, except for $\mathbf{~} \mathbf{B} / \mathbf{c 6}$ for which an exceptional situation was found.

Table S2. Measured pH for PLRP-S mobile phase

\begin{tabular}{lcc}
\hline Buffer & pH, aqueous portion & ${ }^{\mathrm{s}_{\mathbf{w}} \mathbf{p H}, \mathbf{8 0 \%}}$ \\
\hline $0.1 \%$ formate & $\mathbf{A C N}$ \\
$0.1 \%$ Ammonium Acetate & 2.44 & 2.98 \\
$10 \mathrm{mM}$ Triethylamine & 6.74 & 7.69 \\
\hline
\end{tabular}


Figures

Figure S1. PCA scores (A) and loadings (B) plots

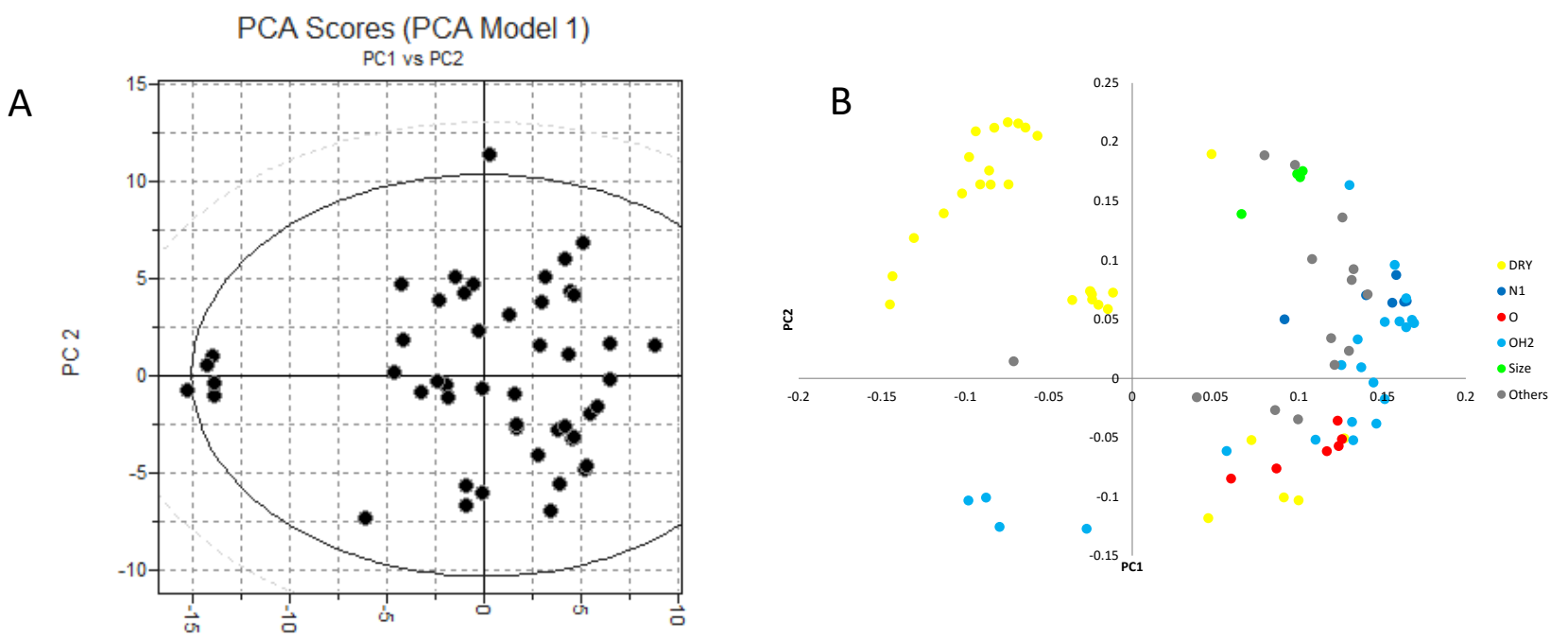


Figure S2. QSPR models external validation

$\log k^{\prime} 80$ PLRP-S

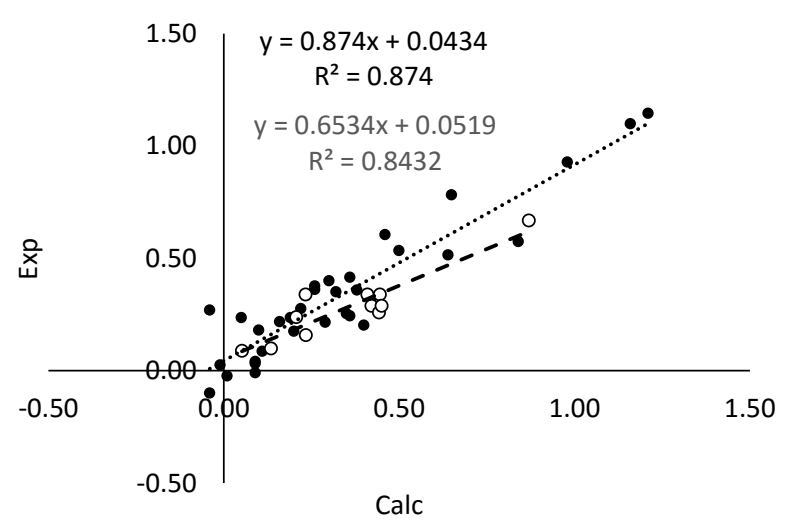

calclog $\mathrm{P}_{\text {tol }}$

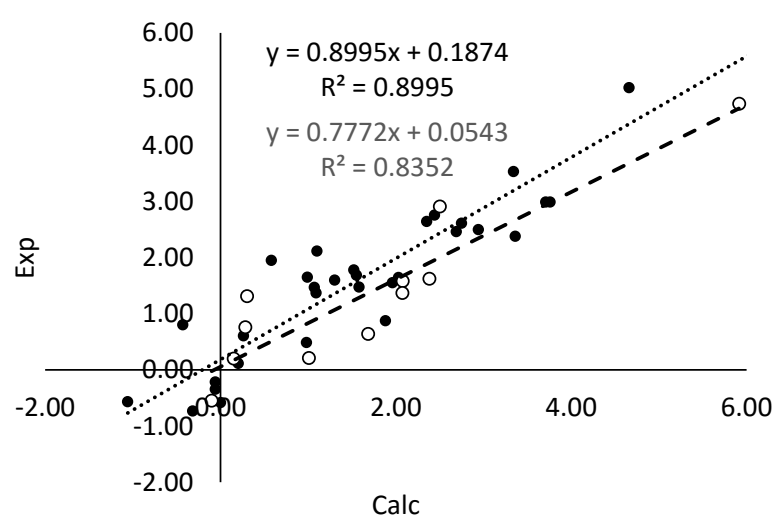

\section{ElogD}

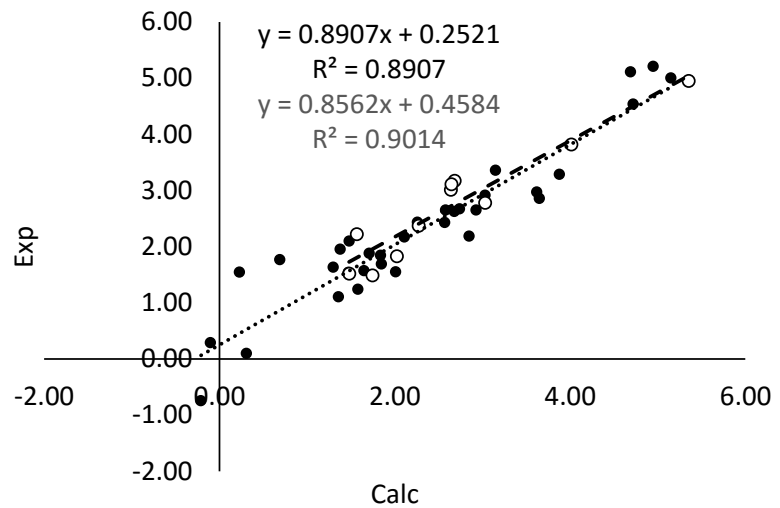

